\title{
Monseigneur Antoine Racine, premier Évêque de Sherbrooke (1874-1893)
}

Jean-Guy Lavallée

Volume 33, 1966

URI : https://id.erudit.org/iderudit/1007319ar

DOI : https://doi.org/10.7202/1007319ar

Aller au sommaire du numéro

Éditeur(s)

Les Éditions Historia Ecclesiæ Catholicæ Canadensis Inc.

ISSN

0318-6172 (imprimé)

1927-7067 (numérique)

Découvrir la revue

Citer cet article

Lavallée, J.-G. (1966). Monseigneur Antoine Racine, premier Évêque de Sherbrooke (1874-1893). Sessions d'étude - Société canadienne d'histoire de l'Église catholique, 33, 31-39. https://doi.org/10.7202/1007319ar

Tous droits réservés @ Les Éditions Historia Ecclesiæ Catholicæ Canadensis Inc., 1967
Ce document est protégé par la loi sur le droit d'auteur. L'utilisation des services d'Érudit (y compris la reproduction) est assujettie à sa politique d'utilisation que vous pouvez consulter en ligne.

https://apropos.erudit.org/fr/usagers/politique-dutilisation/ 


\section{Monseigneur Antoine Racine, premier Evêque de Sherbrooke (1874-1893)}

Nous sommes honorés d'avoir à présenter, devant un auditoire aussi distingué, l'une des plus belles, des plus grandes et des plus nobles figures de l'épiscopat canadien du dernier quart du XIXe siècle, celle de Sa Grandeur Monseigneur Antoine Racine, Evêque fondateur de l'Eglise estrienne dont le règne bienfaisant s'étend de 1874 à 1893 .

Afin de respecter les limites de temps qu'on nous a assignées, nous avons dû nous résoudre à restreindre notre sujet aux fondations les plus importantes que le premier Evêque de Sherbrooke réalisa au cours de son épiscopat. Après avoir brossé, à grands traits, la biographie de Monseigneur Antoine Racine, nous exposerons donc brièvement les événements qui marquèrent la fondation du Séminaire Saint-CharlesBorromée, celle de l'hôpital du Sacré-Cœur et enfin, celle du Collège des Frères. Nous sommes conscients, ce faisant, de mettre de côté une foule d'autres événements importants et de ne pas, par conséquent, donner une idée exacte de la carrière, hélàs trop peu connue, de celui qui fait l'objet de nos recherches.

Avant d'entrer dans le vif de notre sujet, nous voudrions rendre hommage à ceux qui, depuis plusieurs années, ont produit des études auxquelles nous sommes redevables sur Monseigneur Racine et son temps. Dès 1894, année suivant la mort du vénérable fondateur, $M$. l'abbé P.-J.-A. Lefèbvre publiait une notice biographique du premier Evêque de Sherbrooke ${ }^{1}$. Beaucoup plus tard, en 1952, Mgr Albert Gravel consacrait plusieurs pages aux premières années de notre diocèse, dans un ouvrage intitulé : Aux sources de notre histoire religieuse ${ }^{2}$. Enfin, en 1954, M. l'abbé Germain Lavallée produisait une étude des plus sérieuses sur le rôle de Mgr Antoine Racine dans la question universitaire canadienne $^{3}$. Nous nous en voudrions de ne pas mentionner Mgr Maurice 0 'Bready et M. l'abbé Jean Mercier qui ont également beaucoup travaillé à faire connaître notre histoire religieuse. Pour notre part, nos recherches sont encore bien incomplètes et ce n'est qu'en comptant sur votre indulgence que nous avons consenti à vous faire la lecture de ces quelques lignes.

1 L'abbé P. J. A. Lefebvre, Monseigneur Antoine Racine, Premier Evêque de Sherbrooke, Sherbrooke, 1894, B.B.S.

2 Mgr Albert Gravel, Aux sources de notre histoire religieuse, Sherbrooke, Apostolat de la Presse, 1952. L'auteur a également publié plusieurs plaquettes sur l'histoire religieuse de notre région.

3 L'abbé Germain LavalléE, Monseigneur Antoine Racine et la question universitaire (1875-1892), Sherbrooke, 1954. 
Par une bulle datée de Rome, le 28 août $1874^{4}$, Sa Sainteté le Pape Pie IX érigeait, conformément à un vœu de l'Episcopat de la province ecclésiastique de Québec ${ }^{5}$, le Diocèse de Sherbrooke. Quelques jours plus tôt, Messire Antoine Racine, prêtre desservant de l'Eglise Saint-Jean-Baptiste de Québec, avait été prévenu, par une lettre de Rome $^{6}$, qu'il était choisi comme premier Evêque du nouveau diocèse canadien. Une bulle ${ }^{7}$ devait confirmer cette nomination, le premier septembre de cette même année. Une nouvelle Eglise était née, l'Eglise estrienne; elle avait son évêque élu, Sa Grandeur Monseigneur Antoine Racine.

Antoine Racine naquît à Saint-Ambroise-de-la-Jeune-Lorette, dans l'Archidiocèse de Québec, le 26 janvier $1822^{8}$. Son père, Michel Racine, était forgeron, descendant d'une des plus anciennes familles de la côte de Beaupré. Sa mère, Marie Pépin, était la nièce de l'abbé Antoine Bédard, curé de Charlesbourg, prêtre bien connu sous le nom de a Père Bédard *.

C'est chez ce grand-oncle, au presbytère de Charlesbourg, que le jeune Racine commença ses études de latin, durant l'hiver 1833. En 1834, il entra au Petit Séminaire de Québec où, selon le témoignage de l'abbé Lefebvre, son biographe, il fut estimé tant de ses maîtres que de ses condisciples. C'est dans cette vénérable institution qu'il connut l'abbé Jean Holmes qui devait avoir sur lui une influence aussi durable que bienfaisante. C'est également' là qu'il prit le goût de l'étude et de l'assiduité au travail qui le caractérisa toute sa vie. Ses études classiques terminées, Antoine Racine s'orienta vers le Grand Séminaire de Québec.

En étudiant la théologie, comme c'était la coutume à l'époque, le futur Evêque de Sherbrooke, enseigna au Petit Séminaire, son Alma Mater. Le fait d'enseigner en étudiant la théologie ne semble pas l'avoir marqué défavorablement. En effet, Mgr Racine, grand orateur et homme versé dans les sciences divines, semble bien être resté toute sa vie un homme d'étude. Il eut comme confrères de Grand Séminaire des hommes qu'il devait plus tard retrouver au sein de l'Episcopat canadien : Son Eminence le Cardinal Taschereau, NN.SS. Horan, Langevin, Sweenay et McIntyre. Après de brillantes études théologiques, il fut ordonné prêtre le 12 septembre 1844.

4 A.A.S., Bulle d'érection du diocèse de Sherbrooke, 28 août 1874, Insinuations, Vol. I, No 2, p. 3-4.

5 Le Rédacteur du Progrès, Consécration et Intronisation de Mgr Antoine Racine, Premier Evêque de Sherbrooke, Bélanger Frères, Sherbrooke, 1874, p. 10, B.B.S.

6 A.A.S., Lettre signifiant à M. Antoine Racine, prêtre desservant de l'Eglise Saint-Jean-Baptiste de Québec, qu'il est choisi pour être le premier évêque de Sherbrooke, 19 août 1874, Insinuations, Vol. I, N ${ }^{\circ} 1$, p. 2.

7 A.A.S., Bulle nommant M. Antoine Racine, prêtre desservant de l'Eglise Saint-Jean-Baptiste de Québec, premier évêque de Sherbrooke, Insinuations, Vol. I, No 3 , p. 5-7.

8 L'abbé P. J. A. Lefrevre, op. cit. Nous empruntons à cet auteur la plupart des détails biographiques précédant la consécration du premier évêque de Sherbrooke. 
Sa première obédience le conduisit à la Malbaie où il occupa le poste de vicaire. Comme son passé le laissait prévoir, il s'y signala par sa grande bonté, son dévouement inlassable et ses talents d'orateur. A l'exemple de son ancien maître, l'abbé Holmes, et de tant d'autres prêtres de cette génération, il s'intéressa vivement à la colonisation. Sans doute est-ce le zèle qu'il déploya à la faveur de cette grande cause, qui dicta au rédacteur du Progrès du Saguenay, en 1893, l'éloge suivant :

Nous le voyons dès les premières années de son ministère, n'étant que simple vicaire à la Malbaie, visiter lui-même le Saguenay et le Lac SaintJean, et diriger de ce côté les habitants du comté de Charlevoix. De son coup d'œil sûr, il avait vu déjà l'avenir de ces contrées aujourd'hui si florissantes ${ }^{8}$...

En 1848, une nouvelle nomination faisait du jeune abbé Racine le premier curé de Stanfold, aujourd'hui Princeville, dans la région des Bois-Francs. Toujours au témoignage de l'abbé Lefebvre, tout était à faire dans cette nouvelle paroisse. Il n'y avait ni presbytère ni sacristie. Les pauvres colons ne disposaient que d'une chapelle " à peine logeable ». Le nouveau curé se mit à la tâche et organisa convenablement cette paroisse. Là encore, il s'intéressa à la colonisation. Il s'y intéressa au point de rédiger, avec la collaboration d'autres missionnaires de cette région et des Townships, une sorte de manifeste de la colonisation : Le Canadien émigrant. Comme on le remarquera dans les lignes qui suivent, lignes que nous devons à la plume justement admiratrice de l'abbé Lefebvre, son intérêt pour la colonisation ne se limitait pas à la région des Bois-Francs, mais s'étendait à celle des Townships de l'Est :

La vue des misères des braves colons des Bois Francs, les persécutions des grands propriétaires et l'apathie du gouvernement avaient enflammé son patriotisme, et pendant tout le temps qu'il fut à Stanford, il travailla constamment à améliorer la position de ces contrées nouvellement habitées. Ce fut sous son inspiration et sa direction que les douze missionnaires d'alors publièrent Le Canadien émigrant, brochure importante du point de vue de la colonisation, et qui eut alors un grand retentissement. Il adressa aux membres de la Législature des requêtes pressantes, et se transporta même à Toronto, alors le siège du gouvernement, pour les appuyer de sa parole et de son influence; il eut de fréquentes entrevues avec les honorables Lafontaine et Morin, et ne s'éloigna de la Chambre d'Assemblée qu'après avoir obtenu l'assurance de l'Honorable Drummond qu'il se chargerait lui-même de faire passer une loi par laquelle toutes les terres incultes des grands propriétaires contribueraient à l'entretien des chemins et au maintien des écoles 10 .

Malgré son attachement pour nos régions, en 1851, l'abbé Racine devait aller, pour une période d'environ deux ans, exercer son apostolat dans la paroisse de Saint-Joseph de Beauce. Enfin, en 1853, il devenait prêtre desservant de l'église Saint-Jean-Baptiste de Québec, poste qu'il occupait toujours au moment de son élévation à l'épiscopat, comme premier Evêque de Sherbrooke, en 1874.

9 Le Rédacteur du Progrès du Saguenay, cité par l'abbé P. J. A. Lefkbvre, op. cit., p. 45.

10 L'abbé P. J. A. Lefebvre, op. cit., p. 8-9. 
C'est dans l'église paroissiale dont il était pasteur depuis vingt et un ans que Mgr Antoine Racine, Evêque élu de Sherbrooke, reçut la consécration épiscopale des mains de sa Grâce Mgr Elzéar-Alexandre Taschereau, Archevêque de Québec, le 18 octobre $1874{ }^{11}$. Pendant cette mémorable cérémonie, le premier Evêque de Sherbrooke était assisté de NN. SS. LaRocque, Evêque de Saint-Hyacinthe et Langevin, Evêque de Rimouski, tandis que le frère du nouvel évêque, M. le Grand Vicaire Racine de Chicoutimi, agissait comme prêtre assistant ${ }^{12}$. Le sermon de circonstance fut prêché par Mgr de Trois-Rivières. Mgr Laflèche avait choisi, comme texte : "Tu es Petrus...» Il avait parlé, selon le témoignage du Rédacteur du Progrès ${ }^{13}$, qui était présent, pendant plus d'une heure, sur la constitution de l'Eglise catholique, la fonction et le rôle que remplissent le Pape et le corps enseignant des Evêques, pour traiter finalement de l'infaillibilité.

D'après ce même témoin oculaire, plus de sept mille personnes assistaient à cette cérémonie. Parmi celles-là, en plus de l'Evêque consécrateur et des évêques assistants, se trouvaient NN. SS. Persico, Fabre, et Duhamel, Evêque élu d'Ottawa. Environ cent cinquante prêtres étaient présents. De Sherbrooke, MM. les curés Alfred Elie Dufresne de Saint Michel; Chartier de Coaticook; McAuley de Stanstead et Dufresne de Saint-Herménégilde de Barford. La cérémonie avait lieu un dimanche; seuls ces prêtres avaient pu quitter leur paroisse sans que leurs fidèles ne soient privés de la sainte messe ${ }^{14}$. Dans la nef, on remarquait plusieurs membres de la magistrature et du Parlement. Une petite délégation de laïcs du nouveau diocèse s'était rendue à Québec : MM. J. Campbell, M. Conolly, G. E. Rioux et le Rédacteur du Progrès.

L'office fut suivi d'un grand dîner à l'Archevêché de Québec. Dans une touchante allocution qui suivit ce banquet, Monseigneur de Sherbrooke remercia Sa Grâce l'Archevêque de Québec de l'avoir consacré et le Petit Séminaire de Québec pour l'éducation qu'il en avait reçue. Plus tard dans la journée, le nouvel Evêque chanta les vêpres dans l'Eglise basilique Notre-Dame-de-Québec et se rendit ensuite présider une dernière cérémonie dans son église paroissiale. Le nouveau diocèse attendait son Evêque avec impatience.

Le lendemain de sa consécration épiscopale, le 19 août 1874, vers trois heures, Mgr Racine quittait le Palais archiépiscopal de Québec pour s'embarquer sur l'Athenian, mis à sa disposition par la Compagnie Union. Sa Grâce Mgr Taschereau, Son Excellence Mgr Langevin, Sa Grandeur Mgr Fabre et Monseigneur l'Evêque de Saint-Hyacinthe l'accompagnaient, ainsi qu'une centaine de personnes. L'Evêque fondateur de l'Eglise estrienne arriva à Montréal le lendemain vers huit heures, un convoi du Grand Tronc conduisit Mgr Racine et son cortège à

11 A.A.S., Acte de Consécration de Sa Grandeur Mgr Antoine Racine, Premier Evêque de Sherbrooke, 18 octobre 1874, Insinuations, Vol. I, $N^{\bullet} 4$, p. 7.

12 Le Rédacteur du Progrès, op. cit., p. 5.

13 Ibid., p. 6.

14 Ibid., p. 4. 
Sherbrooke. Vers deux heures, une petite manifestation eut lieu à SaintHyacinthe. Un peu plus tard, une collation fut servie à Richmond et enfin, on entra dans la ville épiscopale.

A Sherbrooke, une foule de plus de cinq mille personnes attendait Mgr Racine. Après les cérémonies d'intronisation faites avec le plus de solennité possible, des adresses furent lues l'une au nom du clergé par le Révérend Alfred Elie Dufresne; et une autre au nom des citoyens par le juge Marcus Doherty; et enfin, les Sociétés Saint-Jean-Baptiste et Saint-Patrice y allèrent aussi de leur mot de bienvenue. L'adresse du clergé et celle des citoyens constituent des documents fort intéressants et révélateurs. Après des paroles de bienvenue exprimant la joie commune, on sentit le besoin de dire au nouvel Evêque, la pauvreté du milieu. Mais relisons plutôt M. Dufresne :

Vous ne trouverez ici ni la richesse ni l'organisation que l'on voit dans les diocèses déjà constitués, et ce ne sera que par un long et pénible labeur que vous parviendrez à mettre votre diocèse en état de produire autant de bien que Votre cœur le désire. (...)

Dans le cours de vos visites pastorales, Votre Grandeur trouvera, en quelques endroits, une modeste chapelle où de courageux colons se réuniront. (...) Mais combien d'autres lieux n'auront pas le même bonheur! Les fidèles sans pasteurs n'auront à offrir à Votre Grandeur qu'une pauvre cabane pour y célébrer les saints mystères ${ }^{15}$. (...)

Ainsi parlait à son nouvel Evêque, celui qui, quelques heures plus tard, devait devenir le premier Grand Vicaire du nouveau diocèse. $M$. le juge Doherty lisant l'adresse des citoyens, ne s'exprimait pas autrement :

.... Mais Votre Grandeur connaît d'avance les privations auxquelles l'expose cette transition, et nous espérons qu'elles seront quelque peu adoucies au souvenir des travaux apostoliques de Votre Grandeur dans cette partie du pays à une époque et dans des circonstances encore moins favorables, mais bien propres à qualifier un missionnaire devant remplir plus tard cette haute et importante position $16 . .$.

Le nouveau diocèse n'était donc point riche pour qu'on pense ainsi à en prévenir le nouvel Evêque. N'y avait-il donc pas d'argent dans cette belle région appalachienne de la province de Québec ? La richesse, terre et argent, était concentrée dans les mains de grands propriétaires. Nombre de colons étaient victimes de ces derniers. Cette situation déplorable s'explique par le fait que cette région avait d'abord été ouverte à la colonisation pour les loyalistes et envahie par des immigrants britanniques. Les colons français et catholiques étaient venus sur le tard. Voilà ce qui explique l'état de pauvreté où se trouvaient réduits les fidèles du nouveau diocèse.

Trois diocèses avaient contribué à la formation de celui de Sherbrooke ${ }^{17}$, en cédant un certain nombre de cantons. Saint-Hyacinthe en

15 Le Rédacteur du Progrès, op. cit., p. 9.

16 Ibid., p. Jl.

17 A.A.S., Mandement d'entrée de Mgr Antoine Racine, Premier Evêque de Sherbrooke, Insinuations, Vol. I, $\mathrm{N}^{\circ} 7$, p. 8. 
avait cédé dix-huit, Trois-Rivières vingt-quatre et Québec trois, plus une paroisse. Un peu plus de la moitié de la population de ce territoire n'était pas catholique. En effet, sur une population totale de 68,283 habitants, seulement 30,255 étaient catholiques d'après le recensement de $1870^{18}$. La même année, la future ville épiscopale ne comptait que 2,694 catholiques sur une population totale de 4,432 habitants. Certaines régions, cependant, comptaient plus de catholiques que leurs voisines : le comté de Wolfe, par exemple en comptait 7,886 sur une population totale de 8,823. Mais qu'était-ce, à côté de régions comme les comtés de Richmond. 5,005 sur 11,213; de Compton 4,096 sur 13,665; de Stanstead, 3,497 sur 13,138; de Brôme, 1,848 sur 8,119. Est-ce assez pour montrer que dans plusieurs cantons, la population catholique était en minorité et qu'elle pouvait éprouver certaines difficultés à faire vivre convenablement ses prêtres?

Cette situation ne découragea pourtant pas Mgr Racine. A peine arrivé à Sherbrooke, le 26 octobre 1874, il forme son Conseil épiscopal ${ }^{19}$. Il s'entoure de six prêtres pour que ces derniers l'assistent a de leurs conseils et de leurs lumières \$. Font partie de ce conseil, le révérend Alfred Elie Dufresne, Grand Vicaire et curé de Saint-Michel, les révérends J. B. Chartier; M. McAuley, C. Hamelin, A. Masson et P. Quinn. La première lettre de convocation ${ }^{20}$ adressée aux membres du conseil diocésain nous révèle les premières préoccupations du nouvel Evêque. L'ordre du jour comprend les points suivants :

1. Aviser au moyen de donner au curé ou missionnaire un entretien convenable et au mode à prendre pour y arriver;

2. Etablir un tarif uniforme pour l'Eglise et pour le curé;

3. La fondation du Séminaire;

4. Le nom à donner au nouveau Séminaire 21 .

Certes, le nouvel Evêque avait bien d'autres préoccupations d'ordre spirituel. Il a cependant grand souci d'assurer à tous ses prêtres \& un entretien convenable ». Cette préoccupation n'était pas de trop. En effet, pendant son épiscopat, l'Evêque se verra trop souvent forcé de prévenir les fidèles de plusieurs paroisses qu'ils doivent se soucier de ce devoir. L'établissement d'un tarif uniforme était affaire de discipline qu'il fallait au plus tôt uniformiser, trois parties du diocèse furent soumises à des disciplines différentes. Mais la plus grande préoccupation de Sa Grandeur était de donner un Séminaire à son diocèse, un Séminaire selon le vœu du Concile de Trente. La région avait grand besoin d'une institution comme celle-là, d'un collège classique, mais surtout, il fallait assurer la relève du clergé et donner des prêtres à toutes les parties de l'Eglise estrienne.

18 Le Rédacteur du Progrès, op. cit. Le rédacteur précise, en rapportant ces chiffres, que la population catholique avait augmenté dans certaines parties du nouveau diocèse.

19 A.A.S., Lettre au Rév. A. E. Dufresne, Curé de Saint-Michel de Sherbrooke, le 26 octobre 1874, R.L.E.S., Vol. I, $\mathrm{N}^{\circ}$ 3, p. 2.

20 A.A.S., Lettre au T. Rév. A. E. Dufresne, Sherbrooke, 10 novembre 1874, R.L.E.S., Vol. I, No 10, p. 10-11.

21 A.A.S., Lettre au Rév. A. E. Dufresne, 10 novembre 1874, R.L.E.S., Vol. I, $\mathrm{N}^{\circ} 10$, p. 10-11. 
Il existait déjà, dans la ville de Sherbrooke, depuis 1855, un Institut Littéraire, fondation de Mgr J. C. Prince, premier évêque de SaintHyacinthe. Après l'incendie de l'édifice qui abritait cet institut, en 1857, l'Evêque de Saint-Hyacinthe avait érigé un Collège Industriel où des prêtres et des ecclésiastiques assuraient l'enseignement jusqu'en 1863 pour le céder à des laïcs qui en prirent soin jusqu'en 1875, année de la fondation du Séminaire Saint-Charles-Borromée ${ }^{22}$. Le 2 janvier 1875, Mgr Racine adressait une lettre pastorale au clergé et aux fidèles du diocèse; où il présentait ainsi sa décision :

Tout le diocèse et en particulier la ville de Sherbrooke ont un grand besoin d'un collège commercial et industriel consacré à l'enseignement des enfants et des jeunes gens qui se destinent au commerce et à l'industrie. Dès que les enfants auront terminé leur cours au collège, nous commencerons les classes du Séminaire.

Telle est la résolution que nous avons prise pour rencontrer le vœu général qui demande des études fortes et sérieuses pour le commerce et l'industrie, et dans le but surtout de choisir et de préparer des sujets qui puissent un jour servir l'Eglise naissante de Sherbrooke ${ }^{23}$.

Enfin, le 30 août 1875, l'Evêque de Sherbrooke érigeait par un décret, le Collège Industriel de Sherbrooke en Séminaire diocésain, dont il voulut être le premier supérieur. Le programme comprenait un cours commercial de trois ans et un cours classique de cinq ans. Il est intéressant de constater que les deux langues française et anglaise y étaient enseignées grammaticalement sur un pied d'égalité, ce que l'annuaire justifie de la façon suivante :

La connaissance de ces deux langues devenant de plus en plus importante, particulièrement dans les Cantons de l'Est, il fallait un tel programme pour rencontrer le vœu et, disons-le de suite, pour répondre au besoin de la plus grande partie de la population ${ }^{24}$.

On le voit, les chiffres suffisent à le démontrer, cela était commandé par la situation du temps et ne porte aucune atteinte au patriotisme du premier Evêque de Sherbrooke et de ses prêtres. Mais ce n'était pas une mince tâche de faire vivre et progresser cette nouvelle institution ! L'Evêque dut organiser des quêtes spéciales, il demandait que chaque diocésain sacrifie cinq centins par année à cette fin.

Monseigneur de Sherbrooke accorda beaucoup d'importance à son séminaire. Non seulement il tint à en être le premier supérieur, mais encore, il y enseigna la théologie aux ecclésiastiques pendant plusieurs années, il y versa de ses propres deniers, y donna chaque année un grand nombre de volumes. Au cours de la seule année académique 1875-1876, il donna à la bibliothèque de Saint-Charles, plus de trois cents volumes. Le séminaire était vraiment, comme Mgr Racine se plaisait à le répéter, "l'œuvre la plus utile et la plus nécessaire pour le diocèse ».

22 Annuaire du Séminaire Saint-Charles-Borromée, Sherbrooke, Imprimerie du Séminaire Saint-Charles, année académique 1875-1876, $\mathrm{N}^{\circ} 1$, p. 10-13.

23 Lettre pastorale au clergé et aux fidèles. 2 janvier 1875, dans Annuaire du Séminaire Saint-Charles, année académique 1875-1876, $\mathrm{N}^{\circ} 1, \mathrm{p} .15$.

24 Ibid., p. 6. 
Saint-Charles ne déçut pas le zèle de son fondateur et l'Evêque, malgré l'importance qu'il accordât à son collège, ne s'y limita pas. En 1874, au moment de l'arrivée de Mgr Racine, le diocèse ne comptait que 28 prêtres, 25 chapelles dont cinq à peine convenables. Après dixneuf ans d'épiscopat du premier Evêque, 84 prêtres se dévouaient au bien de l'Eglise estrienne dans 54. paroisses. Le diocèse comptait 60,000 catholiques. L'Eglise était donc solidement implantée en Estrie.

Cette augmentation considérable de la population catholique, dans

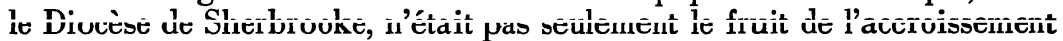
naturel; la colonisation y était aussi pour quelque chose et Mgr Racine avait joué le rôle qui lui revenait dans ce domaine. Il avait aidé, voire inspiré la fondation de la Société de Colonisation de Sherbrooke. Etablie le 14, avril 1880, cette société reçut toute l'aide possible du chef spirituel de la communauté diocésaine. Des quêtes ont été faites dans toutes les églises du diocèse. Monseigneur jugeait cette œuvre nécessaire :

....afin, disait-il, de conserver les éléments constitutifs de notre nationalité contre les efforts que font de puissantes compagnies pour faire occuper par des émigrés européens cette partie du pays que nous habitons ${ }^{25}$.

Voilà bien une preuve du patriotisme authentique et réaliste du premier Evêque de Sherbrooke. Sa Grandeur cherchait en effet à donner à l'Eglise que le Saint-Siège lui avait confiée, des institutions qui durent. Au moment où il travaillait à la fondation de son Séminaire, il voulut venir au secours des malades et des infirmes de la région. Il fonda l'hôpital du Sacré-Coeur. Après avoir acheté un terrain à cette fin, fait construire un édifice, entreprit toutes les démarches, même politiques, qui s'imposaient, l'Evêque attendait les religieuses. Elles arrivèrent le 21 avril 1875, quatre hospitalières de l'Hôtel-Dieu de Saint-Hyacinthe. Les religieuses furent reçues à la gare du Grand Tronc par un grand nombre de Sherbrookois. On les conduisit à la cathédrale où Sa Grandeur leur fit un discours de bienvenue qui traduisait bien sa joie :

Le jour de votre arrivée, mes chères Sœurs, est donc un jour à jamais mémorable pour nous tous, jour de sainte joie, jour de véritable bonheur. (...) Nous savons d'avance au prix de combien de privations et de sacrifices vous accomplirez la sainte mission qui vous est confiée, mais nous savons aussi que, rapportant à Dieu seul le mérite de vos travaux, cet hôpital dont vous prenez aujourd'hui possession resplendira par vous de beauté et de sainteté 26 .

L'hôpital était une œuvre dont' la ville et la région de Sherbrooke avaient grand besoin depuis longtemps. M. le Grand Vicaire Dufresne, alors qu'il était simple curé de Saint-Michel, avait déjà fait des démarches en vue de l'établissement de cette institution. C'est à la puissante personnalité de Mgr Racine que cette fondation était réservée. En effet, l'Evêque avait su rapidement convaincre tout le monde, y compris les autorités civiles provinciales, de la nécessité de cette fondation au cœur de l'Estrie.

25 A.A.S. Biographies des prêtres du diocèse de Sherbrooke.

26 Annuaire du Séminaire Saint-Charles-Borromée, année académique 1882 $1883, \mathrm{~N}^{\bullet} 8$, p. 57. 
Le Séminaire Saint-Charles-Borromée, tout en assurant la relève du clergé, pouvait pourvoir à la formation des futurs hommes d'affaire. L'hôpital du Sacré-Cœur, pour sa part, apportait aux malades l'espoir de la guérison et aux infirmes, un asile. Il restait un problème capital à régler : celui de l'école élémentaire catholique et française pour garçons. Précisément à cause de la présence de deux groupes ethniques différents par la langue et la foi, les catholiques de langue française étant en minorité, l'éducation élémentaire des garçons était mal assurée. Jusqu'en 1853, la situation fut des plus pénibles. Bien des parents refusaient d'envoyer leurs enfants à l'école parce qu'il était presque impossible de les faire bénéficier de l'enseignement de la religion et du français. Après 1853, l'élection de commissaires catholiques et français devait améliorer la situation. Pourtant, il fallait faire davantage, assurer cette éducation catholique et française par la présence de religieux éducateurs. Cette tâche aussi revint à Mgr Racine. Au cours de l'été 1882, on construisit, dans le quartier du Centre, une nouvelle école. Le 26 novembre 1882, l'Evêque lui-même en faisait la bénédiction solennelle et profitait de l'occasion pour souhaiter la bienvenue aux Frères du Sacré-Cœur :

... C'est donc de grand cœur que nous accueillons dans notre diocèse les vénérés Frères du Sacré-Cœur, ces puissants auxiliaires du clergé auprès des enfants qu'ils instruisent par leurs sages leçons, qu'ils édifient par les les exemples d'une vie toute faite d'abnégation et de charité 27.

Bien qu'il restât plusieurs réalisations à accomplir, l'Evêque était presque comblé. L'éducation chrétienne et française des garçons était assurée. Mgr Racine ne s'arrêta pas là pour autant. Mais l'essentiel était réalisé. Comme l'écrit fort justement l'abbé Lefèbre;

Le Séminaire, l'Hôpital et le Collège des Frères sont les trois auvres principales de Mgr Racine dans sa ville épiscopale 28.

A la fin de cet exposé, nous éprouvons un sentiment de culpabilité et d'injustice à l'endroit du vénérable fondateur de l'Eglise estrienne. C'est, en effet, être bien incomplet, que de réduire l'œuvre de ce grand homme à quelques fondations. Ce faisant, nous laissons dans l'ombre plusieurs aspects de sa personnalité et combien de ses ouvres les plus chères. Si l'implantation solide de l'Eglise en Estrie a été l'œuvre de sa vie, elle ne s'est pas limitée, loin de là, à ce que nous en avons dit. Ajoutons que pas un seul des problèmes capitaux de son époque n'a laissé Mgr Racine indifférent, au contraire, il faut convenir qu'il a largement contribué à la solution de plusieurs difficultés. Nous aurions voulu souligner ses interventions dans les problèmes que posaient la question universitaire, les affaires d'influences indues, le vote des catholiques, les oppositions parfois vives entre libéraux et ultramontains. Nous aurions alors découvert, dans cet humble évêque de province, non seulement le colonisateur, le fondateur, mais aussi, l'orateur, le patriote, et avant tout, l'homme de Dieu et le serviteur de l'Eglise universelle.

Jean-Guy LAVALlÉE

Professeur d'Histoire, Université de Sherbrooke

27 Ibid., p. 57.

28 L'abbé P. J. A. Lefrbvre, op. cit., p. 17. 